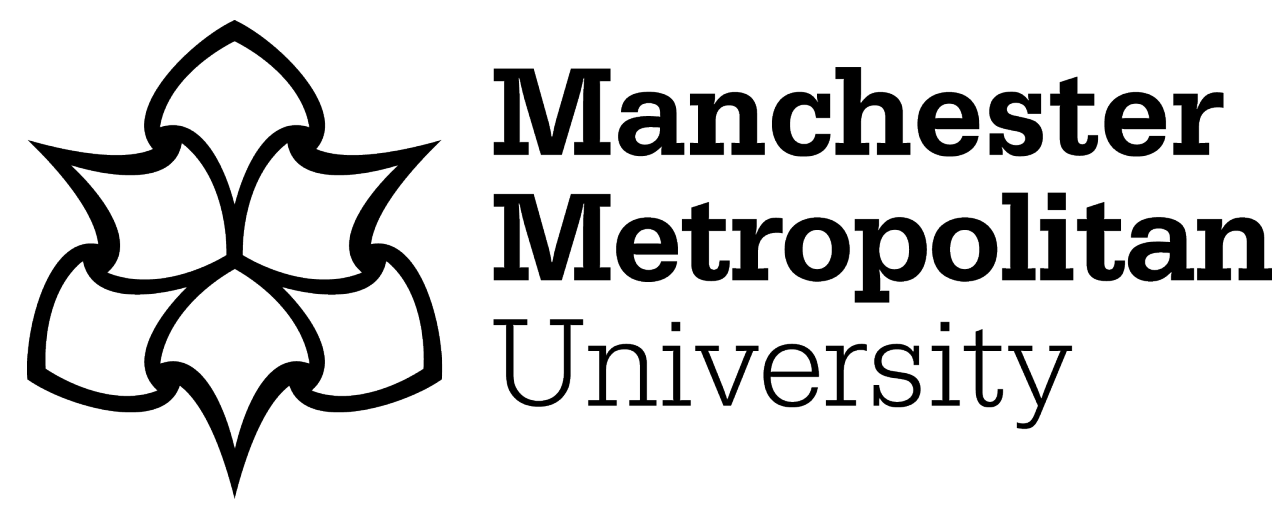

Blackhurst, EJ and Salmon, NA (2020) Detection of concealed explosives and shrapnel weapons using decompositions of microwave polarimetric radar data. In: Millimetre Wave and Terahertz Sensors and Technology XIII, 21 September 2020 - 25 September 2020, Online.

Downloaded from: https://e-space.mmu.ac.uk/626700/

Version: Accepted Version

Publisher: SPIE

DOI: https://doi.org/10.1117/12.2565953

Please cite the published version 


\title{
Detection of concealed explosives and shrapnel weapons using decompositions of microwave polarimetric radar data
}

\author{
Eddie J. Blackhurst ${ }^{\text {a) }}$, Neil A. Salmon ${ }^{\text {b) }}$, a) School of Physics and Astronomy, The University of \\ Manchester, Jodrell Bank, SK11 9DL, UK. b) Faculty of Science and Engineering, Manchester \\ Metropolitan University, All Saints, Oxford Road, Manchester, M15 6BH, UK.
}

\begin{abstract}
Surrogate explosives and shrapnel weapons at a range of 2 metres have been measured using a full polarimetric radar operating over the band 18-26 GHz. Measurements of these items were made as they were standing by themselves and as they were placed on the body, under light clothing. These measurements were compared with measurements made of the divested human body. The polarimetric radar comprised a vector network analyser, and orthomode transducer and a waveguide conical horn antenna. The measurements were analysed using the Euler/Huynen decompositions and the Cloude/Pottier decompositions working on the coherency matrix, as derived from multiple time sequence measurements. The results conclude that the signature of threat items changes considerably when they are placed on the human body. The measured signature of the threat item on the body appears to be somewhere between that of the threat item when it is by itself and that of the divested human body.
\end{abstract}

Keywords: Concealed weapons, decomposition, Monostatic, millimetre wave, explosives, Huynen fork, coherency matrix, Sinclair matrix.

\section{INTRODUCTION}

Development of microwave sensing technology has developed over many decades to become highly mature, while technology costs continue to fall. The transparency of clothing has enabled this technology to be exploited in many different ways for the detection of weapons concealed under clothing. Over the past 40 years, the decomposition techniques of polarimetric radar have evolved to become highly effective in identifying different areas of land in remote sensing from aerial platforms. This area of work combines the monostatic polarimetric radar decomposition techniques with microwave sensing technology to explore the opportunities for stand-off security screening of people for concealed threats, building on previous work [1] [2] [3].

\section{DECOMPOSITIONS}

The decomposition techniques of polarimetric radar process the measured calibrated $2 \times 2$ Sinclair backscattering matrices to identify key signatures of targets. Decompositions fall into two basic categories, coherent techniques and incoherent techniques. Coherent decomposition techniques operate directly on a single Sinclair matrix. In a monostatic configuration, the Sinclair matrix is complex and symmetric, so data contained is represented by only six real numbers. Assuming one of these numbers is the phase from the radar to the target, the Sinclair matrix is considered as holding five pieces useful real numbers associated with the target.

Examples of coherent decompositions are the Pauli and Krogager methods [4]. The Pauli decomposition reduces the Sinclair matrix into a plane reflector and dihedral reflectors at orientations of $0^{\circ}$ and $45^{\circ}$. The Krogager decomposition reduces the matrix into a plane reflector, a dihedral reflector and a helical reflector. However, these are limited as they cannot identify dipole reflectors uniquely. For example, dipole scatterers in these decompositions appear as a combination of plane and dihedral reflectors.

If a target does not change in itself the Sinclair matrix may still change via movements and interactions (reflections) with its environment, including the radar. This phenomenon is referred to as depolarisation and in the scenarios of this paper, it is almost the same type of phenomenon as speckle. Fortunately, the incoherent decomposition methods provide some means of extracting target information in the presence of depolarisation, even though the Sinclair matrix may be changing its component values as a function of time. 
Incoherent decomposition techniques operate directly on the coherency matrix. In a monostatic radar configuration, the coherency matrix is a $3 \times 3$ Hermitian matrix formed by summations of the Kronecker product of the linearised Sinclair matrix with its complex conjugate. Summations can be made over the radiation frequency or multiple sweeps of the VNA over the full band, or both. In these measurements of this paper, the summation was made over the sweeps, so the decompositions could be observed as a function of radiation frequency. Being a Hermitian matrix, the coherency matrix contains nine real numbers. Since this is greater than the five real numbers of the Sinclair it potentially carries more information for the identification of targets.

Cloude/Pottier decomposition is [4], [5] an example of an incoherent decomposition technique. By extracting the eigenvalues and eigenvectors of the coherency matrix, the information is reduced to three parameters, the entropy $(H)$, the scattering parameter $(\alpha)$ and the anisotropy $(A)$. Entropy appears to be a measure of the amount of movement and random scattering from the target. The alpha is a discriminator that indicates the dominant type of scattering, this being, $0^{\circ}-30^{\circ}$ for plane reflectors, $30^{\circ}-60^{\circ}$ for dipoles and $60^{\circ}-90^{\circ}$ for dihedrals. It cannot however uniquely identify helical reflectors.

The Huynen Fork and the Huynen/Euler (HE) target parameters are [6] [7] [8] a decomposition technique that associates a target with its physical characteristics. There are five HE parameters generated, these being the maximum in the polarimetric radar cross-section amplitude $(m)$, its roll related orientation $(\phi)$, the number of bounces radar radiation undergoes on reflection $(v)$, its ability to polarise radar radiation $(\gamma)$ and the target symmetry $(\tau)$. These parameters can be derived directly from the Sinclair matrix [9] or the coherency (or Kennaugh) matrix [10]. When targets are stationary both methods generate identical results for the HE parameters. When targets are moving the coherency matrix route generates the more accurate results about the target properties [11]. For the Huynen Fork presented in this paper, the HE target parameters were calculated from the coherency matrix.

Further useful metrics for analysis are the target smoothness, roughness and depolarisation, which can all be extracted from the Kennaugh (or coherency) matrix [12].

\section{MEASUREMENTS OF EXPLOSIVES AND SHRAPNEL WEAPONS}

The polarimetric radar used in these measurements comprises a vector network analyser (VNA), an orthomode transducer (OMT) [13] and a conical waveguide horn antenna, measuring a subject at a range of 2 m over an 18-26 GHz radiation bandwidth and a $10 \mathrm{kHz}$ intermediate frequency bandwidth, as described in [1]. There were 801 equally spaced measurement frequencies over the radiation band and it took the system about 1 second to measure and record each sweep of this frequency range. For measurements of the coherency matrix, the number of sweeps was between nine and one hundred. The $3 \mathrm{~dB}$ antenna width at the $2 \mathrm{~m}$ range was about $40 \mathrm{~cm}$ in diameter. The data was calibrated using a large area ( $75 \mathrm{~cm}$ across) plane reflector and a dihedral angled at $45^{\circ}$ using the method described in [1], with range gating to reject clutter in both the calibration and target acquisition measurements.

The decompositions are investigated for two types of target. The first is a surrogate shrapnel weapon ( $31.5 \mathrm{~cm}$ long by 12 $\mathrm{cm}$ wide by $2.2 \mathrm{~cm}$ thick) comprising a collection of galvanised steel felt nails contained in a paraffin wax binder. The second is a slab of beeswax, $3.8 \mathrm{~cm}$ thick, chosen as a surrogate explosives weapon, as beeswax is an accurate simulant of a class of nitrogen-based energetic materials [14]. The results of the decompositions are shown separately for the measurements of: a) the weapons by themselves, b) the human torso with no threats present, c) the surrogate threat items on the human torso. In the cases of the human torso, the person was standing as motionless as possible, with no intentional body movement, and with all personal items such as phones, watches and belts etc being divested. The measurement region of the body was at the front in the centre of the abdomen.

\subsection{Surrogate explosives and shrapnel on a foamed polystyrene stand}

The measurement of completely stationary targets on foamed polystyrene stand is useful, as this can then be compared with how they look when they are on the body of a person. Foamed polystyrene has a low coefficient of reflection and in the calibration procedure, the reflection from the stand is subtracted as background from the target measurements.

\subsubsection{Surrogate explosives - beeswax slab}

Measurements of the $3.8 \mathrm{~cm}$ thick beeswax block are shown in Figure 1 and illustrate some of the key features of this stationary target. For targets that are not moving, successive measurements of the components of the Sinclair matrix are essentially identical as illustrated in the figure. Furthermore, the appearance of the Huynen Fork in the Poincaré Sphere over the radiation band $18-26 \mathrm{GHz}$ is a horizontal circle along the equator for the cross-minima, with the co-minima at the 
top and bottom circular polarisation poles. This indicates that the reflecting object is plane surface like across the whole frequency band. This is also substantiated by the Pauli and Krogager decompositions indicating the dominant response is that of a plane surface. The smoothness indicates the surface was indeed very smooth, with a roughness to smoothness ratio of $1 \times 10^{-3}$. The entropy with its very low value of $3.7 \times 10^{-4}$ indicated the surface was stationary and the alpha of $\sim$ $3^{\circ}$ indicated the surface was plane. It can also be noted that the entropy is very closely matched to the depolarisation. The above measurements also show a very regular variation in the metrics with frequency and this is the result of constructive and destructive interference in the etalon cavity formed by the flat parallel surfaces of the beeswax, as reported in [1].
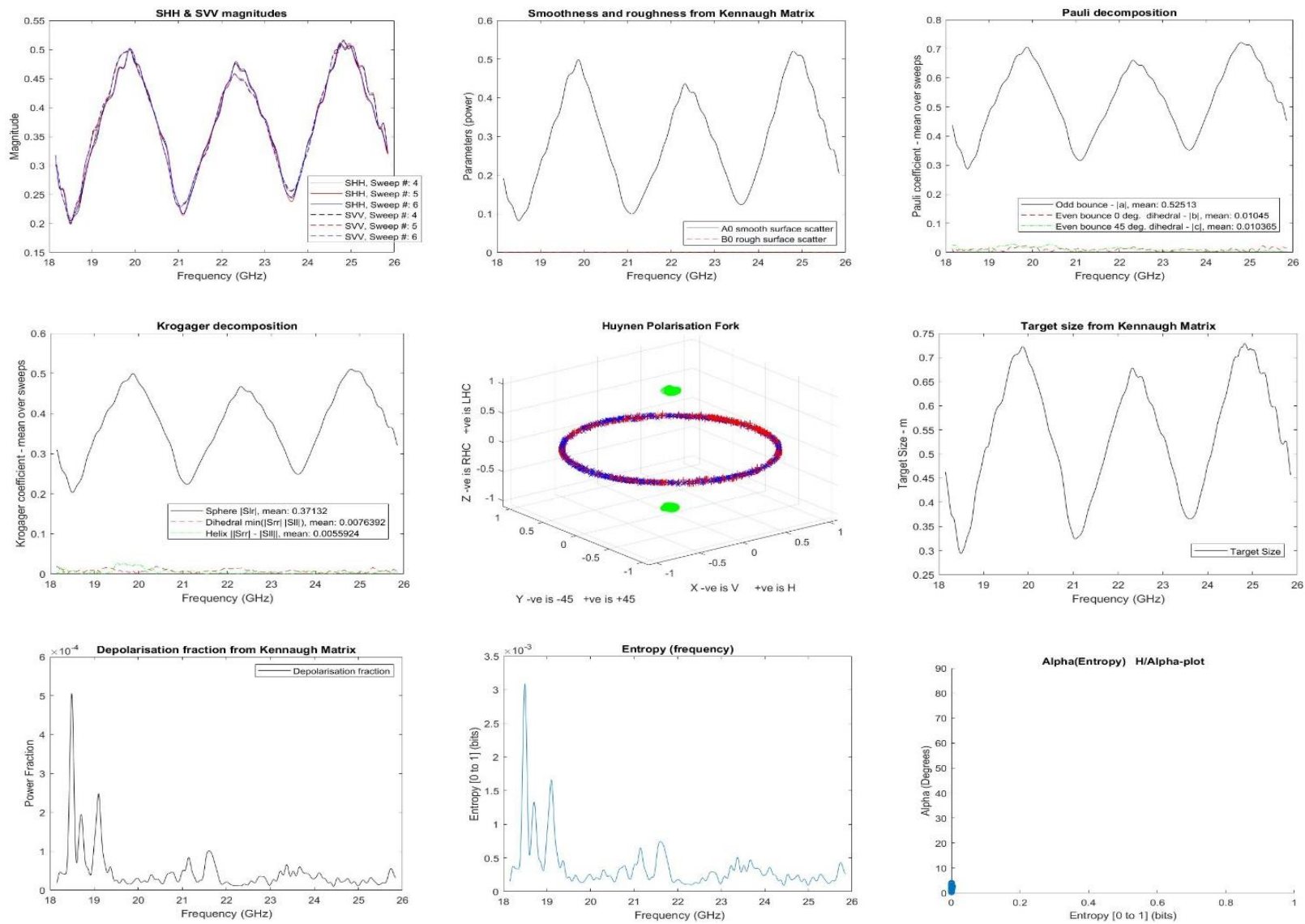

Figure 1. For the beeswax surrogate energetic material shown here from the top left to the bottom right are the Sinclair parameters, the Huynen Parameters (smoothness: black, roughness: red), the Huynen Fork (black: cross-minimum, red: sub-cross-minimum, green: co-minima), the target size, the smoothness, the Pauli decomposition (black: plane, red: $0^{\circ}$ dihedral, green: $45^{\circ}$ dihedral), the Krogager decomposition (black: plane, red: dihedral, green: helical), depolarisation (blue) and the Cloude/Pottier decomposition (entropy and alpha: both black). This colour key is that same in the following figures, but for brevity is not repeated there.

\subsubsection{Surrogate shrapnel weapon - nails in paraffin wax slab}

Similar measurements of the shrapnel weapons also indicate key information about the target. It can be seen that the smoothness of the shrapnel weapon is much less than that of the beeswax and that the roughness is much greater, with a roughness to smoothness ratio of 0.33 . The Pauli and Krogager decompositions indicate that the target is much less of a plane reflector, but a combination of other components. The Huynen Fork shows a large variation in the coordinates of the cross-minima and co-minima over the frequency band. The entropy of the Claude/Pottier decomposition shows a low value of $6.7 \times 10^{-4}$, indicating little physical movement. The alpha spans $5^{\circ}$ to $65^{\circ}$ indicating there is a spread in the types of scattering, from plane type surface reflectors to dipole type reflectors. The decompositions are showing the target to be something that is making a quasi-random scattering of the incident radar illumination, which is what might be expected from the disordered array of the nails in the paraffin wax. 

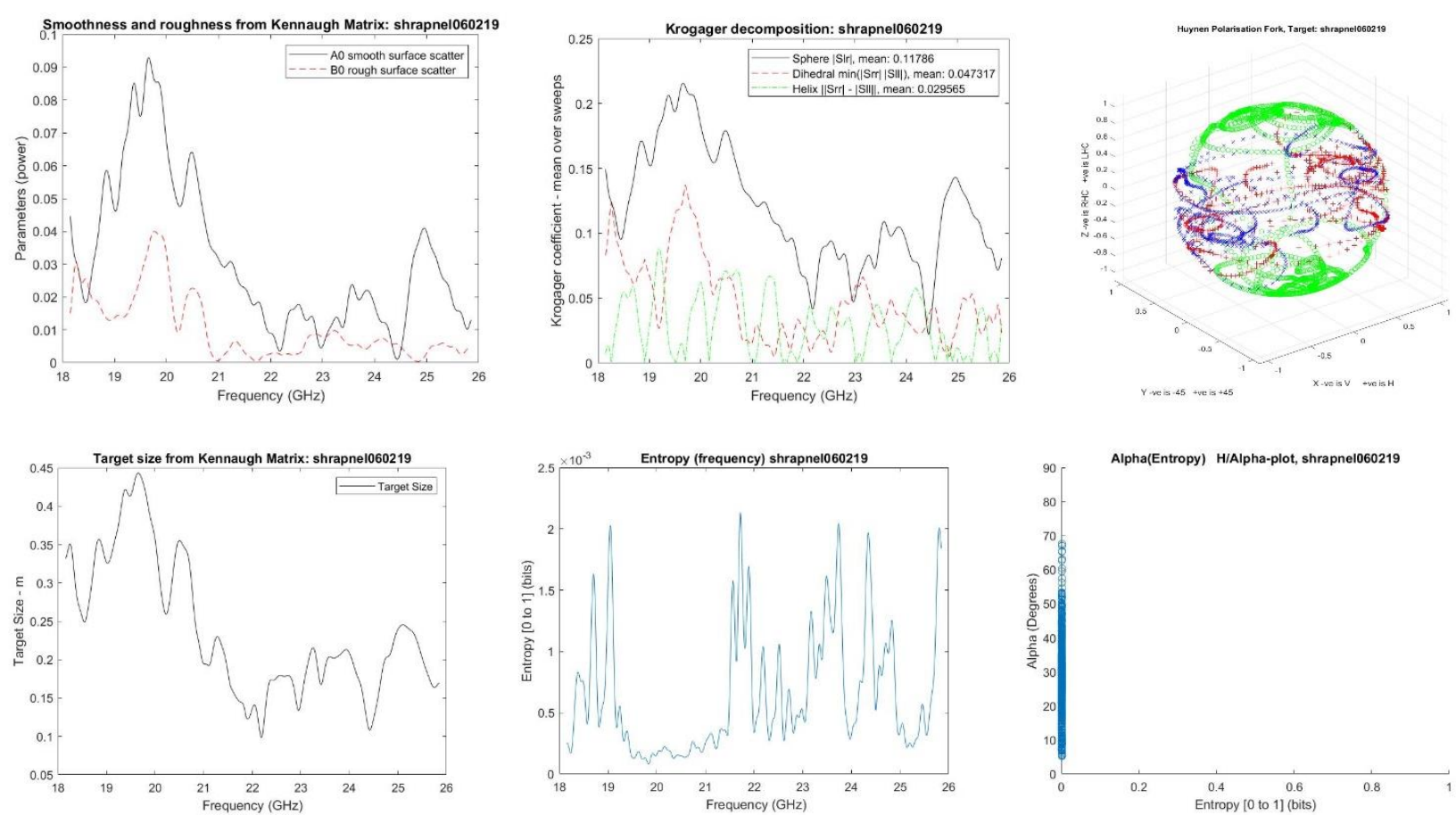

Figure 2. For the shrapnel weapon shown here from the top left to the bottom right are the smoothness, the Pauli decomposition, the Krogager decomposition, the Huynen Fork, the target size and the Cloude/Pottier decomposition.

\subsection{The human torso divested}

Measurements of the human torso alone, with no weapon, are also presented for comparison in Figure 3. The Sinclair parameters from three consecutive measurements indicate substantial changes between each measurement. These changes arise from the very small involuntary movements of the body, for example breathing. The mean roughness to smoothness ratio over the band was estimated at $\sim 0.18$, which is far greater than that of the smooth-surfaced beeswax and half that of the shrapnel weapon. The Pauli and Krogager decompositions indicate the plane surface reflection to be the dominant mode, but with other dihedral scattering mechanisms at about $30 \%$ of this. The Huynen Fork indicates the target to be plane surface like but with deviations in the cross-minima from the equator and deviations of the co-minima from the circular polarisation poles. The mean entropy over the frequency band was estimated to be 0.41 , which is far greater than that of a completely motionless target, this higher value arising from the small involuntary body movements. The alpha across the frequency band ranges from $10^{\circ}$ to $30^{\circ}$ indicating it to be closest to a plane reflector type, but with some characteristics of dipole reflectors. These measurements are typical of a divested person. 

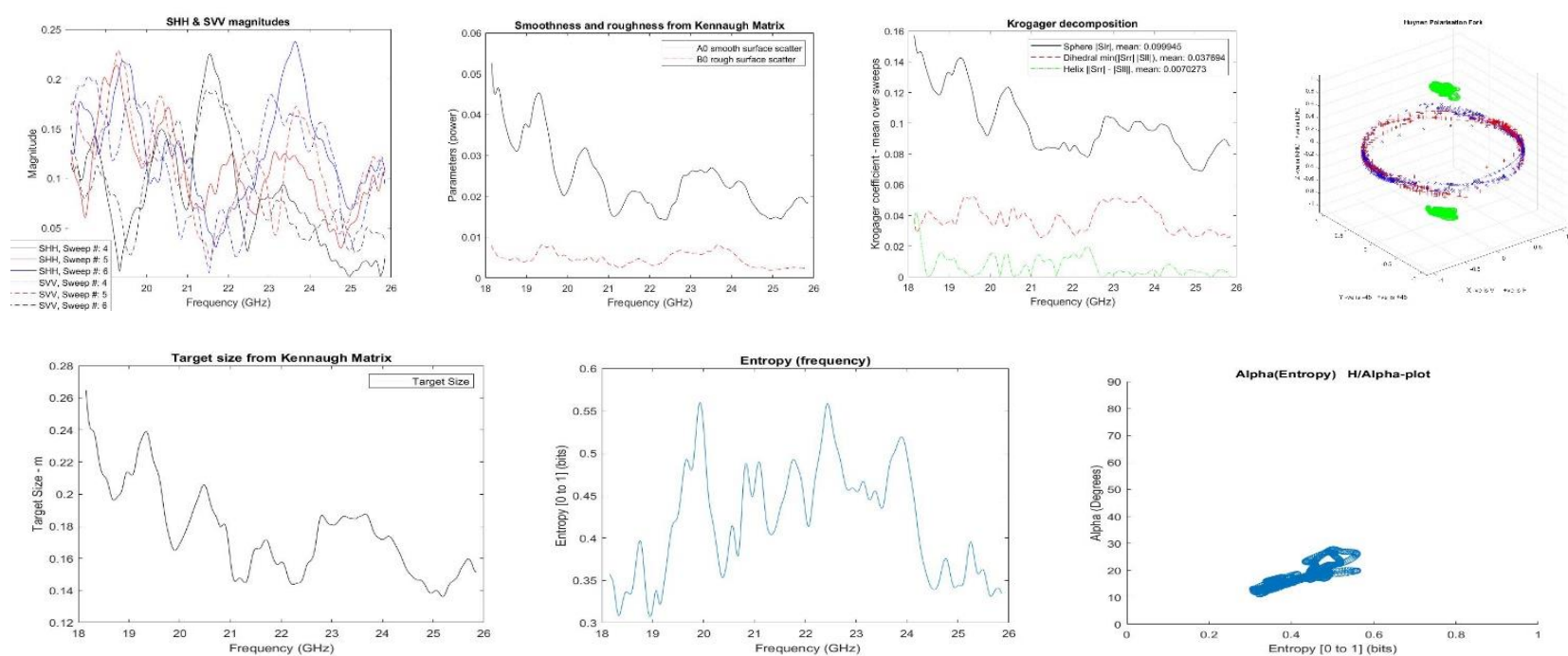

Figure 3: From the top left to the bottom right the Sinclair parameters, the smoothness, the Pauli decomposition, the Krogager decomposition, the Huynen Fork and Entropy and Alpha from the Cloude/Pottier decomposition.

\subsection{Surrogate explosives and shrapnel weapons on the human torso}

\subsubsection{Beeswax block on the human torso}

Measurements of the beeswax block on the human torso are shown in Figure 4. The mean roughness to smoothness ratio of $\sim 0.14$ indicates a value slightly less than that of the beeswax on its own. The Pauli and Krogager decompositions indicate the dominant scattering mechanism is still that of the plane surface. The Huynen Fork indicates a slight deviation from a plane surface by the spread of the cross-minima about the equator and the movement of the co-minima away from the circular polarisation poles. The deviation from a plane surface like response is greater than that deviation for the divested human torso. The entropy from the Cloude/Pottier decomposition has a mean value of 0.27 over the band, which is consistent with a level associated with human involuntary movement. The alpha scattering parameter extends from $7^{\circ}$ up to $35^{\circ}$ which is similar to that of the human torso by itself.
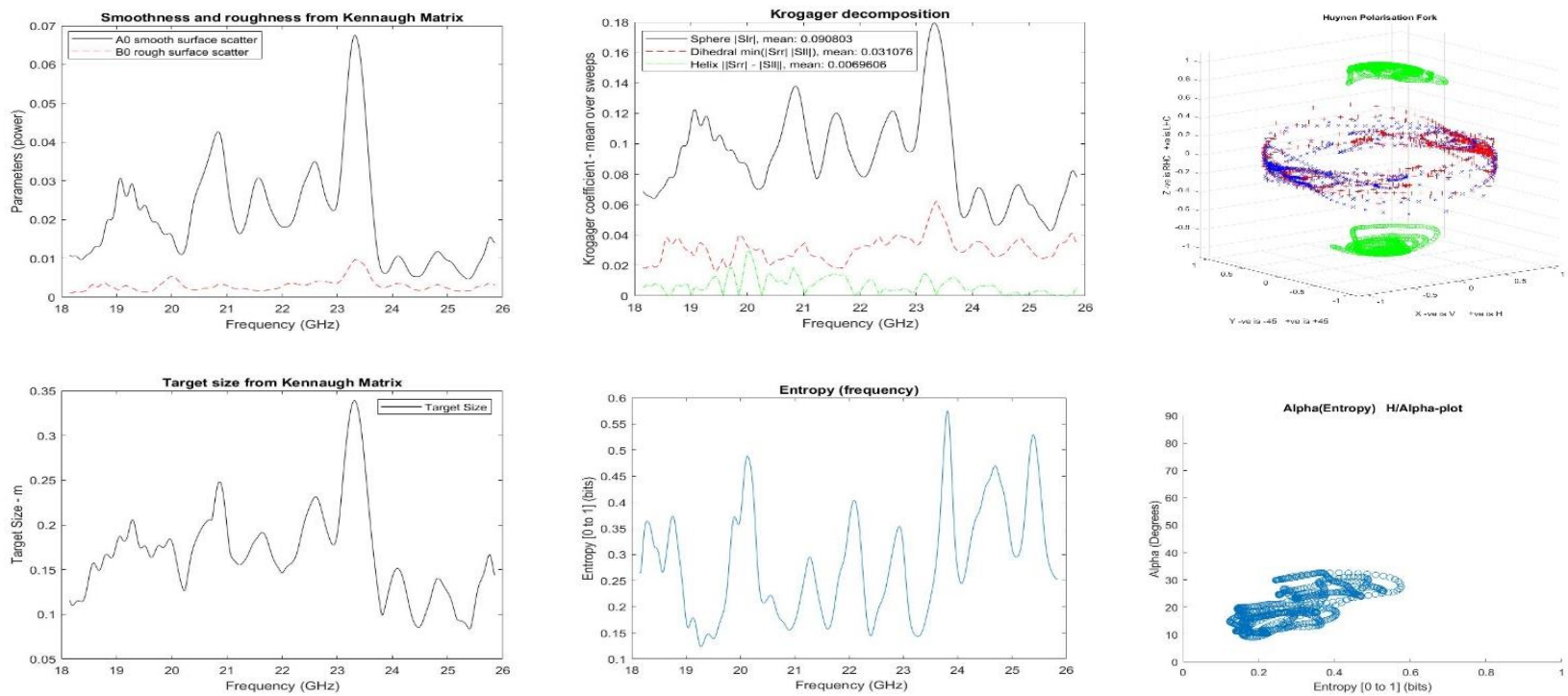

Figure 4. For the beeswax on the body shown are the smoothness, the Paul and Krogager decompositions, the target size, the Huynen Fork and the Cloude/Pottier entropy and scattering parameter. 


\subsubsection{Steel nails in paraffin wax against the human torso}

Measurements of the shrapnel on the body indicate the mean roughness to smoothness ratio is 0.29 . The Pauli and Krogager decompositions indicate the dominant reflection type is the plane reflector, but the target size shows a mean value of 0.368 , which is higher than that of the human body alone. The Huynen Fork indicates a slightly greater deviation from a plane reflector than the beeswax against the body. Some of the transitions of the cross-minima and co-minima in the Poincare Sphere in these types of targets show similarities between when they are on their own and when they are on the human torso [11]. The entropy is high with a mean value of 0.54 , greater than that for the human body alone.
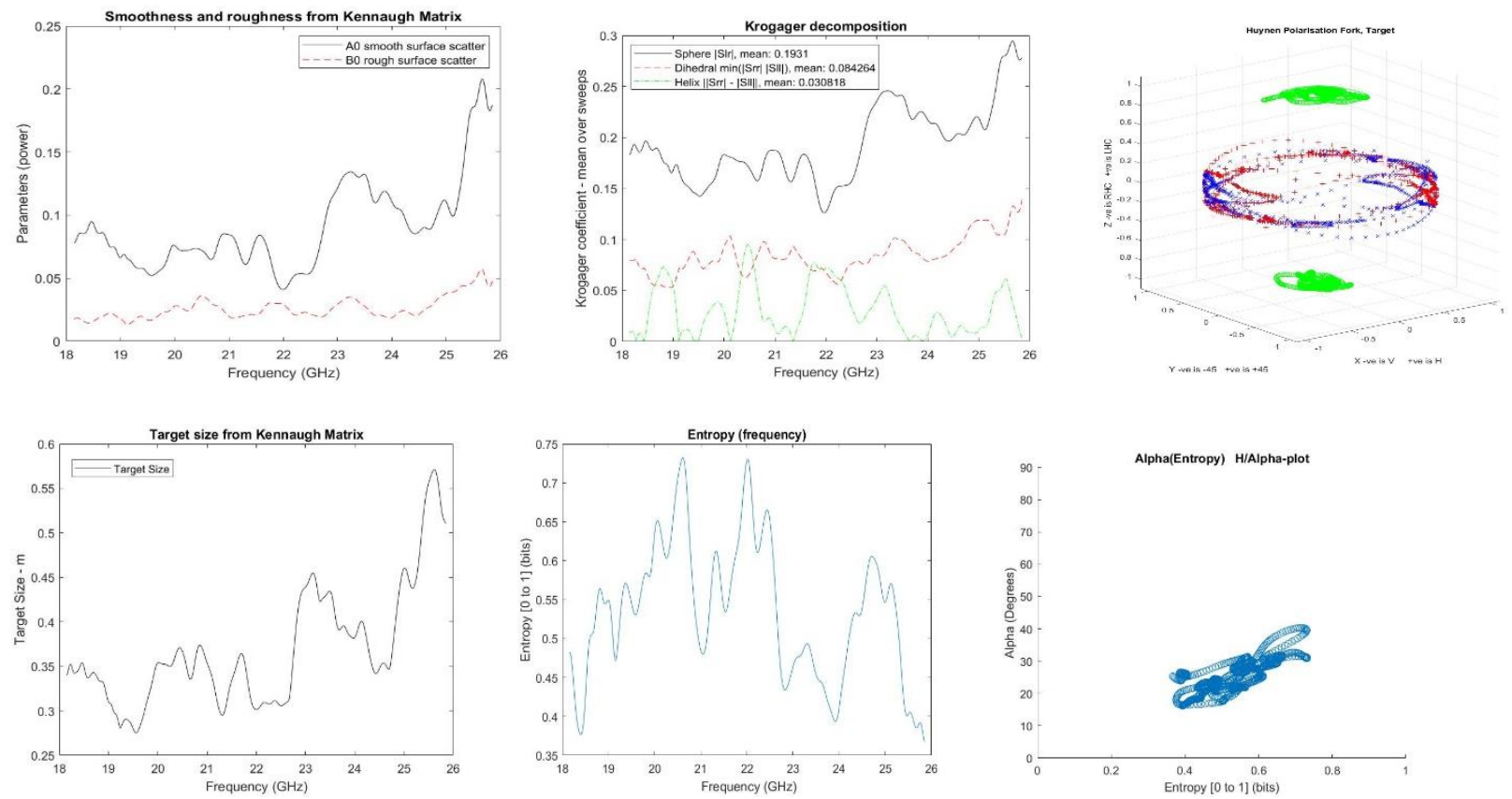

Figure 5. Measurements of the shrapnel weapon against the human body, showing the smoothness, the Pauli and Krogager decompositions, the Huynen Fork, the target size and the entropy and scattering parameter.

\section{CONCLUSION}

Full polarimetric radar measurements have been made of surrogate explosives and shrapnel weapons as they are standing by themselves on a foamed polystyrene stand and when they are against the human body. For comparison, the divested human body has also been measured. A slab of beeswax as a surrogate explosive has been found to have characteristic features of regular variation of target cross-section with frequency, whilst still looking like a plane reflector, from the nature of the Huynen Fork. However, when the material was placed on the human body, the regular variations in target radar cross-section changed and the Cloude/Pottier entropy was greatly increased. When the shrapnel weapon was measured by itself, there were higher roughness to smoothness ratios and there were large variations in the HE parameters with frequency, as can be seen in the Huynen Fork plot. The Cloude/Pottier entropy however, was similarly low to that of the beeswax measured by itself. When the shrapnel weapon was placed against the body there was seen to be less variation in the HE parameters as can be seen from the Huynen Fork plot and the entropy has greatly increased. The overall conclusion of this work is that the decompositions are revealing the physical characteristics of surrogate explosives and weapons. However, the understanding of the relationship between decompositions and the targets needs to be developed further if it is to be used for threat recognition.

\section{FUTURE WORK}

It is intended that more and varied types of threat materials be measured and that the benefits of operating at lower and higher radiation frequencies should be considered. Lower frequencies may enable operation at close ranges, these typically being a few wavelengths, whilst higher frequencies (up to $300 \mathrm{GHz}$ ) may enable longer-range performances. Decompositions investigated in this paper are those developed for remote sensing. Efforts will be made to develop decomposition techniques that are specific to screening for concealed weapons and threats. 


\section{REFERENCES}

[1] E. Blackhurst and N. A. Salmon, "Full polarimetric radar for concealed weapons detection: Experimental determination and simulation of the Huynen target parameters for the human torso," Proc. SPIE 11164, Millimetre Wave and Terahertz Sensors and Technology XII, vol. 11164, p. 111640D, 2019, doi: 10.1117/12.2547833.

[2] E. Blackhurst, N. Salmon and M. Southgate, "Experimental determination and simulations of the Huynen target parameters for full polarimetric millimetre wave concealed weapon recognition," Proc. SPIE 10800, Millimetre Wave and Terahertz Sensors and Technology XI, vol. 10800, p. 1080007, 2018, doi: 10.1117/12.2324450.

[3] E. Blackhurst, N. Salmon and M. Southgate, "Full polarimetric millimetre wave radar for stand-off security screening," Proc. SPIE 10439, Millimetre Wave and Terahertz Sensors and Technology X, vol. 10439, p. 1043906, 2017, doi: 10.1117/12.2282564.

[4] S. Cloude, Polarisation - applications in remote sensing, Oxford University Press, 2010.

[5] E. Keydel, E. Pottier, M. Hellmann, S. Cloude and L. Ferro-Famil, Radar Polarimetry and Interferometry, Brussels, Washington and Ottawa: NATO, 2004.

[6] J. Huynen, Phenomenological theory of radar targets, $\mathrm{PhD}$ dissertation, Drukkerij Bronder, Rotterdam, The Netherlands: Delft University of Technology, 1970.

[7] A. Xi and W. Boerner, "Determination of the characteristic polarization states of the radar target scattering matrix [S(AB)] for the coherent monostatic and reciprocal propagation space by using the complex polarisation ratio $\rho$ transformation formulation," Opt. Soc. Am. A, vol. 9, no. 3, pp. 437-455, 1992.

[8] W. Boerner, W. Yan and A. Xi, "On the Basic principles of Radar Polarimetry: Target Characteristic Polarisation State Theory of Kennaugh, Huynen's Polarization Fork Concept, and its Extension to the Partially Polarized Case," Proceedings of the IEEE, vol. 79, no. 10, pp. 1538-1550, 1991.

[9] A. Agrawal and W. M. Boerner, "Redevelopment of Kennaugh's Target Characteristic Polarisation State Theory Using the Polarization Transformation Ratio Formalism for the Coherent Case," IEEE Transactions on Geoscience and Remote Sensing, vol. 27, no. 1, pp. 2-14, 1989.

[10] C. Baird, W. T. Kersey, R. Giles and W. E. Nixon, "Classification of targets using optimized ISAR Euler imagery," in Defence and Security Symposium, Radar Sensor Technology X, Radar Sensor Technology X; Proc. Vol. 6210, Kissimmee, 2006, doi: 10.1117/12.664964.

[11] E. J. Blackhurst, Development of techniques and technology for full polarimetric radar applied to concealed weapons detection, $\mathrm{PhD}$ doctoral dissertation, Manchester Metropolitan University, 2020.

[12] J.-S. L. Lee and E. Pottier, Polarimetric Radar Imaging - from basics to applications, Boca Raton: CRC Press, 2009.

[13] A. Navarrini and R. L. Plambeck, "A Turnstile Junction Waveguide Orthomode Transducer," IEEE Transactions on Microwave Theory and Techniques, vol. 54, no. 1, pp. 272-277, 2006.

[14] N. A. Salmon, "Indoor Full-Body Security Screening: Radiometric Microwave Imaging Phenomenology and Polarimetric Scene Simulation," IEEE Access, vol. 8, pp. 144621-144637, 2020, doi: 10.1109/ACCESS.2020.3013967. 